\title{
CALIDAD DE PITAHAYA AMARILLA (Selenicereus megalanthus) EN DIFERENTES ESTADOS DE MADUREZ Y TEMPERATURAS DE CONSERVACIÓN
}

\section{QUALITY OF YELLOW PITAHAYA (Selenicereus megalanthus) IN DIFFERENT STATES OF MATURITY AND STORAGE TEMPERATURES}

Annabell Eloísa Vera Vera ${ }^{1}$, Yenny Alexandra López Vera ${ }^{1}$, Saskia Valeria Guillen Mendoza ${ }^{1}$, Sofía del Rocío Velásquez Cedeño ${ }^{1}$, Cristian Fernando Chila Chila ${ }^{1}$

\begin{abstract}
${ }^{1}$ Carrera de Ingeniería Agrícola, Escuela Superior Politécnica de Manabí Manuel Félix López, calle 10 de agosto ํo 82 y Granda Centeno, Calceta, Manabí, Ecuador.

Email:sguillen@espam.edu.ec

\section{Información del articulo}

Tipo de artículo: Nota técnica

Recibido:

$25 / 01 / 2021$

Aceptado:

Resumen

En la presente investigación se evaluó el efecto de tres estados de madurez (inicial, media y completa) sobre las propiedades físicoquímicas de los frutos de pitahaya amarilla (Selenicereus megalanthus), en dos temperaturas de conservación (zonas cálidas $26,8^{\circ} \mathrm{C}$ y controlada $20^{\circ} \mathrm{C}$ ). Las variables registradas fueron: peso del fruto $(\mathrm{g})$, diámetro $(\mathrm{mm})$, firmeza $\left(\right.$ newtons $\left./ \mathrm{cm}^{2}\right)$, sólidos solubles $\left(\right.$ grados Brix $\left.{ }^{\circ}\right), \mathrm{pH}$ y acidez titulable. Los datos se sometieron a un análisis de varianza y separación de medias con prueba de Tukey al 5\%. El estado de madurez inicial a temperatura controlada presentó la menor reducción de peso $(125,04 \mathrm{~g}), \mathrm{pH}(4,18)$ y mayor reducción de la acidez $(17,0)$ y firmeza $\left(6,0 \mathrm{~N} / \mathrm{cm}^{2}\right)$ a excepción del diámetro $(57,5 \mathrm{~mm})$ y sólidos solubles $\left(16,9 \mathrm{Brix}^{\circ}\right)$ que presentaron mejores resultados en los frutos cosechados a madurez completa en temperatura controlada.
\end{abstract}

$10 / 12 / 2021$

Licencia:

CC BY-NC-SA 4.0

Revista ESPAMCIENCIA 12(2):141-151

DOI:

https://doi.org/10.5

1260/revista_espam ciencia.v12i 2.233
Palabras clave: Pitahaya amarilla, estados de madurez, temperaturas, calidad postcosecha.

\begin{abstract}
In the present investigation, the effect of three stages of maturity (initial, medium and complete) on the physicochemical properties of the fruits of yellow pitahaya (Selenicereus megalanthus) was evaluated, in two storage temperatures (warm zones $26.8^{\circ} \mathrm{C}$ and controlled $20^{\circ} \mathrm{C}$ ). The variables recorded were: fruit weight $(\mathrm{g})$, diameter $(\mathrm{mm})$, firmness (newtons $/ \mathrm{cm}^{2}$ ), soluble solids (degrees Brix $\left.{ }^{\circ}\right), \mathrm{pH}$ and titratable acidity. The data was subjected to an analysis of variance and separation of means with the Tukey test at $5 \%$. The initial state of maturity at controlled temperature presented the least weight reduction $(125.04 \mathrm{~g}), \mathrm{pH}(4.18)$ and the greatest reduction in acidity (17.0) and firmness $\left(6.0 \mathrm{~N} / \mathrm{cm}^{2}\right)$ except diameter $(57.5 \mathrm{~mm})$ and soluble solids $\left(16.9 \mathrm{Brix}^{\circ}\right)$ that presented better results in the fruits harvested at full maturity at controlled temperature.
\end{abstract}

Keywords: Yellow pitahaya, maturity stages, temperatures, postharvest quality.

\section{INTRODUCCIÓN}

La pitahaya (Selenicereus megalanthus), es una planta rústica de la familia cactácea, es conocida mundialmente como "la fruta del dragón". El fruto presenta características físicas y químicas de acuerdo a la especie, pueden ser diversas como: la presencia de espinas, color de piel y de la pulpa, sólidos solubles y pH (Jiménez et al. 2017). Esta fruta tiene gran potencial industrial a nivel mundial debido a su alto contenido de propiedades y son considerados una gran ventaja para la agroindustria lo cual ha incrementado el interés internacional por este cultivo para la comercialización como para la búsqueda de alternativas y procesamientos de diversos productos, sin embargo las perspectivas del mercado internacional requieren de investigaciones que 
lleven a elevar la calidad de la fruta, genotipos élite para la siembra y manejo en la cadena productiva (Montesinos et al. 2015).

La pitahaya amarilla cultivada en Ecuador es vista como una nueva opción de producción agrícola, elevando el nivel de vida de los agricultores; con el fin de desplegar una mayor diversificación en el mercado internacional, para dar a conocer la pitahaya como una fruta con alto potencial de comercialización e industrialización (Aguilar, 2015).

Ecuador dispone aproximadamente de 1528 hectáreas de pitahaya con un rendimiento promedio de 7,6 t/ha. En la Amazonía ecuatoriana, específicamente en la provincia de Morona Santiago la transición rápida de pitahaya (ecotipo "Palora") de planta silvestre a cultivo comercial ha provocado problemas de manejo agronómico y postcosecha (Vargas et al., 2020).

Son pocas las frutas que se conocen y forman parte la dieta diaria, sin embargo, la variedad de estos alimentos es muy amplia, entre los frutos que han tomado un realce en la última década se nombra a la pitahaya (Huachi et al. 2015).

La información que existe en el país es escasa especialmente en la provincia de Manabí sobre la calidad de esta fruta, sin embargo, estudios realizados han determinado que los frutos de pitahaya ecotipo Palora maduros presentan valores altos en firmeza, acidez titulable, sólidos solubles, $\mathrm{pH}$, vitamina $\mathrm{C}$, capacidad antioxidante y polifenoles totales; siendo la mayor cantidad de fruta exportable con mínimas pérdidas postcosecha (1,5\%) (Sotomayor et al. 2019).

Además, se destaca que la vida útil de la pitahaya amarilla es relativamente corta, para su consumo en fresco o procesado, por lo cual se deben desarrollar investigaciones que ofrezcan alternativas de conservación para mejorar la calidad y el tiempo en percha, permitiendo así que la pitahaya mantenga su sabor, aroma y su peculiar efecto laxante (Dueñas et al. 2009).

Los parámetros utilizados en investigaciones para determinar la calidad de la fruta son: peso total del fruto, peso de la cáscara (pericarpio), peso de la pulpa y semilla, sólidos solubles totales (SST) en el jugo, $\mathrm{pH}$ y acidez titulable (relación ${ }^{\circ} \mathrm{Brix} / \mathrm{Acidez}$ ), porcentaje de azúcares totales, Vitamina $\mathrm{C}$, contenido de fenoles totales, capacidad antioxidante (DPPH) y color (Balderas et al., 2016)

Entre los factores del manejo tras la cosecha, los más importantes son grado de madurez al momento de la cosecha, temperatura, humedad relativa, composición de la atmósfera (Magaña et al., 2013).
En estudios realizados por Sotomayor et al. (2019) el fruto pesa aproximadamente $331.6 \mathrm{~g}$ y cuenta con un diámetro polar en estados de cosecha temprano de 82 . $\mathrm{mm}$ y en estados tardíos de $75.3 \mathrm{~mm}$ y una longitud de $12 \mathrm{~cm}$ del fruto de y un diámetro en épocas tempranas de $82.9 \mathrm{~mm}$ y tardías $75,3 \mathrm{~mm}$.

Garcia et al. (2008), describe que la firmeza en escala de 0 a 6 en estados de madurez es generalmente es de 17,77 $\mathrm{N}$ y $6,20 \mathrm{~N}$ y que la cantidad de sólidos solubles totales obtenidas en época inicial del fruto es de $20.1^{\circ}$ Brix. Mientras que la acidez titulable en época temprana de cosecha es de 0,12 en época tardía es de 0,10 , además el $\mathrm{pH}$ es de 4,4 a 4,7 en época de cosecha temprana y en madurez de 3,7 a 4,8

Con base en los estudios anteriores y considerando que existen pocos reportes de este tipo de estudio en la provincia de Manabí, la investigación tuvo el objetivo de evaluar el efecto de tres estados de madurez inicial (1), medio (3) y completo (5) (figura 2), sobre las propiedades físicoquímicas de los frutos de pitahaya amarilla (Selenecereus megalanthus) en dos temperaturas de conservación (ambiente $26,8 \mathrm{C}^{\circ}$ y controlada $20^{\circ} \mathrm{C}$ ).

\section{MATERIALES Y MÉTODOS}

\section{Localización}

Los frutos utilizados para este ensayo fueron cosechados en la hacienda EL OKASO, localizada en el sitio La Papaya perteneciente al cantón Rocafuerte de la provincia de Manabí, ubicada geográficamente en las coordenadas $0^{\circ} 52^{\prime} 48^{\prime \prime}$ latitud Sur y $80^{\circ} 23^{\prime} 02^{\prime}$ " longitud Oeste, a una altitud entre $17 \mathrm{msnm}$. La investigación se realizó en los meses de enero y febrero de 2020, en el Laboratorio de postcosecha de la Escuela Superior Politécnica Agropecuaria de Manabí Manuel Félix López.

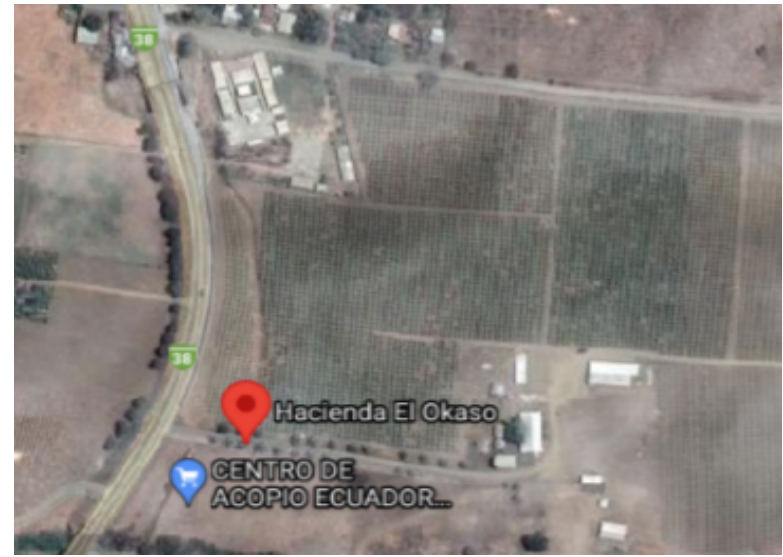

Figura 1. Mapa de la ubicación de la hacienda EL OKASO

Fuente: Google Maps, 2020 


\section{Clasificación de frutos}

Los frutos se cosecharon en el mes de enero del 2020 y se trasladaron en gavetas al laboratorio de postcosecha de la Escuela Superior Politécnica Agropecuaria de Manabí, en donde fueron clasificados con base en los estados de madurez de acuerdo a la carta de color (figura 2 ). Se escogieron frutos en los grados 1,3 y 5 (madurez inicial, media y completa).

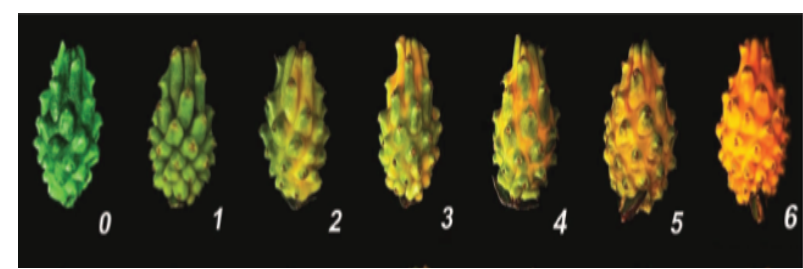

Figura 2. Carta de color de pitahaya amarilla (Selenecereus megalanthus)

Fuente: Guerrero (2014)

\section{Diseño y unidad experimental}

Se utilizó un diseño completamente al azar con arreglo factorial A x B, en donde el factor A corresponde al grado de madurez y el factor $\mathrm{B}$ corresponde a la temperatura en la que se realizó el experimento, teniendo como resultado seis tratamientos (Cuadro 1), se realizaron cuatro réplicas para cada tratamiento, las cuales estuvieron conformadas por 15 frutos por réplica. Los datos se registraron a los $0,3,6,9$ y 12 días después de la cosecha, en cada evaluación se destruyeron 3 frutos al azar de cada réplica para el registro de variables químicas obteniendo 15 frutos por tratamiento para cada evaluación y para las físicas se evaluó los mismos tres frutos por réplica a los $0,3,6,9$ y 12 días.

Cuadro 1. Descripción de tratamientos para el ensayo calidad de pitahaya amarilla (Selenicereus megalanthus) en diferentes estados de madurez y temperaturas de conservación

\begin{tabular}{|c|c|}
\hline $\begin{array}{c}\mathrm{N}^{0} \text { de } \\
\text { tratamientos }\end{array}$ & Descripción \\
\hline 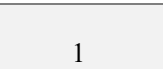 & $\begin{array}{l}\text { Madurez Inicial + Temperatura Ambiente } 26,8 \mathrm{C}^{\circ} \\
\text { (MI+TA). }\end{array}$ \\
\hline 2 & $\begin{array}{l}\text { Madurez Media + Temperatura Ambiente } 26,8 \mathrm{C}^{\circ} \\
(\mathrm{MM}+\mathrm{TA}) .\end{array}$ \\
\hline 3 & $\begin{array}{l}\text { Madurez Completa + Temperatura Ambiente } 26,8 \\
\mathrm{C}^{\circ} \text { (MC+TA). }\end{array}$ \\
\hline 4 & $\begin{array}{l}\text { Madurez Inicial + Temperatura Controlada } 20 \mathrm{C}^{\circ} \\
(\mathrm{MI}+\mathrm{TC}) .\end{array}$ \\
\hline 5 & $\begin{array}{l}\text { Madurez Media }+ \text { Temperatura Controlada } 20 \\
\mathrm{C}^{\circ}(\mathrm{MM}+\mathrm{TC}) .\end{array}$ \\
\hline 6 & $\begin{array}{l}\text { Madurez Completa }+ \text { Temperatura Controlada } 20 \\
\mathrm{C}^{\circ}(\mathrm{MC}+\mathrm{TC}) .\end{array}$ \\
\hline
\end{tabular}

\section{Análisis de datos}

Los datos de las evaluaciones se sometieron a un análisis de varianza y separación de medias con prueba de Tukey al 5\%, utilizando el programa estadístico INFOSTAT versión 2019.

\section{Análisis físicos}

Para la evaluación del peso, diámetro y firmeza se escogieron los respectivos frutos a evaluar y se procedió con el registro de las variables ya mencionadas. Para obtener el peso se utilizó una balanza digital (Shimadzu ELB3000, Japón), con una sensibilidad de 0,1 g. Para la variable diámetro se utilizó un calibrador vernier (Stainless Helios Hardened Throughout, Alemania). La firmeza fue registrada con un durómetro no destructivo (Turoni, 53215TT, escala 0-100 Shore, Italia) cada tres días.

\section{Análisis químicos}

Para las variables sólidos solubles, $\mathrm{pH}$ y acidez titulable, se evaluaron 12 frutos por tratamiento. Para medir el contenido de solidos solubles totales se colocó una gota en un refractómetro manual ATAGO ${ }^{\circledR}$ (Japón), expresando los sólidos solubles totales en grados Brix. El pH se tomó ubicando el jugo de cada fruta en un vaso de precipitación al cual se le introdujo un electrodo marca Oakton 700, Estados Unidos. Para medir la acidez titulable se colocó $50 \mathrm{~mL}$ del jugo en una alícuota al cual se le añadió 3 gotas de fenolftaleína (indicador), y por medio de una bureta con $\mathrm{NaOH} \mathrm{0,1} \mathrm{N} \mathrm{(titulador)} \mathrm{se} \mathrm{agitó}$ hasta alcanzar un cambio de color (rosado purpura), para esto se empleó el método de la Association of Official Analytical Chemist (AOAC, 1998).

\section{RESULTADOS Y DISCUSIÓN}

\section{Pérdida de peso (g)}

El peso del fruto presentó una reducción lineal en el tiempo, independientemente de la temperatura y los estados de madurez estudiados (cuadro 2). De acuerdo con el modelo de regresión lineal el mayor ritmo de pérdida de peso se dio en el estado de madurez completa en temperatura controlada con 1,84 g, mientras que en los estados de madurez inicial y media en temperatura controlada fue de 1,81 y 1,51 g/día; en temperatura ambiente la mayor pérdida fue en el estado de madurez media con 1,64 gr, a diferencia de la madurez inicial y completa con 1,61 y 1,61 gr, respectivamente. Resultados similares de Sotomayor et al. (2019) en pitahaya amarilla obtuvieron diferencias estadísticas significativa para la variable peso de fruto similares a esta investigación, además mencionan que el peso fresco de la fruta aumenta conforme avanzan los distintos estados fenológicos. Otros resultados reportados por Jiménez et al. (2017), quienes no observaron diferencias significativas en pérdida de peso para frutos de pitahaya amarilla entre los diferentes tratamientos en cuanto a las variables de temperatura, reportaron que al ambiente (promedio $17^{\circ} \mathrm{C}$ ) los frutos perdieron un promedio del $12.5 \%$ de peso y en temperatura controlada $\left(6 \pm 2^{\circ} \mathrm{C}\right)$ mostraron una pérdida 
promedio del $8.2 \%$ en los tres estados de madurez. Por lo consiguiente, Alvarado (2014) observó diferencias estadísticas significativas en pitahaya amarilla, demostrando que durante la maduración postcosecha existe una reducción de peso de entre 9 y $10 \mathrm{~g}$ entre cada periodo de evaluación de la fruta. Verificando que sí existe una diferencia de peso de 49,6 g desde que la fruta ha sido cosechada hasta 15 días después, correspondiendo al $16,98 \%$ del peso de la fruta.

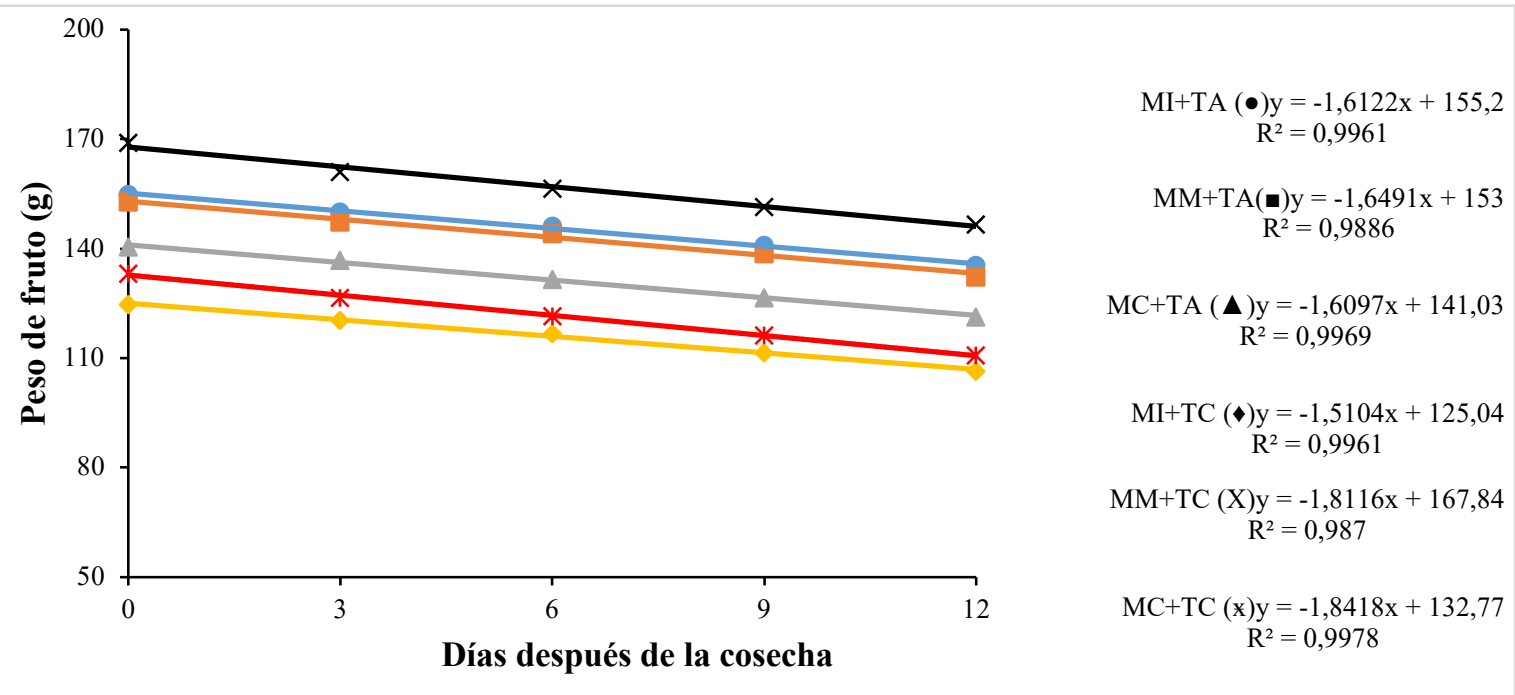

Gráfico 1. Pérdida del peso en frutos de pitahaya amarilla cosechados en tres estados de madurez, bajo condiciones de temperatura ambiente y controlada

Cuadro 2. Medias de peso de fruto (gr) de pitahaya amarilla (Selenicereus megalanthus), letras diferentes en el día de evaluación representan separación de medias significativas de acuerdo al test de Tukey al 95\% de confianza.

\begin{tabular}{|c|c|c|c|c|c|c|}
\hline \multirow{2}{*}{\multicolumn{2}{|c|}{ Tratamiento }} & \multicolumn{5}{|c|}{ Peso de fruto (gr) } \\
\hline & & Día 0 & Día 3 & Día 6 & Día 9 & Día 12 \\
\hline \multicolumn{2}{|c|}{$\mathrm{MI}+\mathrm{TA}$} & $155 \mathrm{ab}$ & $150,1 \mathrm{ab}$ & $146,21 \mathrm{ab}$ & $140,97 \mathrm{ab}$ & $135,36 \mathrm{ab}$ \\
\hline \multicolumn{2}{|c|}{$\mathrm{MM}+\mathrm{TA}$} & $152,8 \mathrm{ab}$ & $147,34 \mathrm{ab}$ & $144,23 \mathrm{ab}$ & $138,75 \mathrm{ab}$ & $132,39 \mathrm{ab}$ \\
\hline \multicolumn{2}{|c|}{$\mathrm{MC}+\mathrm{TA}$} & $140,5 \mathrm{ab}$ & $136,75 \mathrm{ab}$ & $131,59 \mathrm{ab}$ & $126,61 \mathrm{ab}$ & $121,42 \mathrm{ab}$ \\
\hline \multicolumn{2}{|c|}{$\mathrm{MI}+\mathrm{TC}$} & $124,75 \mathrm{~b}$ & $120,38 \mathrm{~b}$ & $116,689 \mathrm{~b}$ & $111,58 \mathrm{~b}$ & $106,49 \mathrm{~b}$ \\
\hline \multicolumn{2}{|c|}{$\mathrm{MM}+\mathrm{TC}$} & $169,1 \mathrm{a}$ & $161,03 \mathrm{a}$ & $156,55 \mathrm{a}$ & $151,57 \mathrm{a}$ & 146,64 a \\
\hline \multicolumn{2}{|c|}{$\mathrm{MC}+\mathrm{TC}$} & $133,2 \mathrm{ab}$ & $126,61 \mathrm{ab}$ & $121,64 \mathrm{ab}$ & $116,33 \mathrm{ab}$ & $110,76 \mathrm{ab}$ \\
\hline \multicolumn{2}{|c|}{ Probabilidad } & $0,015^{*}$ & $0,0195^{*}$ & $0,0141^{*}$ & $0,0272 *$ & $0,0232 *$ \\
\hline Factores & Niveles & & & & & \\
\hline \multirow{3}{*}{$\begin{array}{l}\text { Estados de } \\
\text { madurez (A) }\end{array}$} & Inicial & $139,87 \mathrm{~b}$ & $135,23 \mathrm{ab}$ & $131,45 \mathrm{ab}$ & $126,27 \mathrm{ab}$ & $120,93 \mathrm{ab}$ \\
\hline & Medio & 160,9 a & 154,18 a & $150,39 \mathrm{a}$ & 145,17 a & $139,52 \mathrm{a}$ \\
\hline & Completo & $136,87 \mathrm{~b}$ & $131,68 \mathrm{~b}$ & $126,62 \mathrm{~b}$ & $121,47 \mathrm{~b}$ & $116,09 \mathrm{~b}$ \\
\hline \multirow{2}{*}{$\begin{array}{l}\text { Temperatura } \\
\text { (B) }\end{array}$} & Ambiente & 149,44 & 144,72 & 140,68 & 135,44 & 129,73 \\
\hline & Controlada & 142,36 & 136,01 & 131,63 & 126,49 & 121,30 \\
\hline \multirow{2}{*}{ Probabilidad } & Factor A & $0,0177^{*}$ & $0,0258^{*}$ & $0,0167^{*}$ & $0,0289^{*}$ & $0,027^{*}$ \\
\hline & Factor B & $0,3056 \mathrm{NS}$ & $0,2022 \mathrm{NS}$ & $0,1727 \mathrm{NS}$ & $0,2134 \mathrm{NS}$ & $0,2293 \mathrm{NS}$ \\
\hline \multicolumn{2}{|c|}{ C.V (\%) } & 11,28 & 11,48 & 11,47 & 12,98 & 13,21 \\
\hline
\end{tabular}




\section{Diámetro del fruto $(\mathrm{mm}$}

El diámetro del fruto presentó un comportamiento ligeramente descendente en las dos temperaturas y los diferentes estados de madurez (cuadro 3 ). En el gráfico 2 se observa que el diámetro disminuyó 0,$62 ; 0,66$ y 0,71 $\mathrm{mm}$ en los frutos con madurez inicial, media y completa, respectivamente, en temperatura ambiente; mientras que en temperatura controlada la reducción fue de $0,65 \mathrm{~mm}$ en madurez inicial, $0,63 \mathrm{~mm}$ en madurez media y 0,60 mm en madurez completa. Para García y Robayo (2008) que evaluaron el uso de atmósferas modificadas pasivas y temperaturas bajas en la conservación de pitahaya amarilla evidenciaron una reducción del diámetro en un 6 a 8\%. En la Norma NTE-INEC-025 se describe que los frutos de pitahaya amarilla que son aptos para ser comercializados en mercados internacionales oscilan entre 8 y $12 \mathrm{~cm}$.

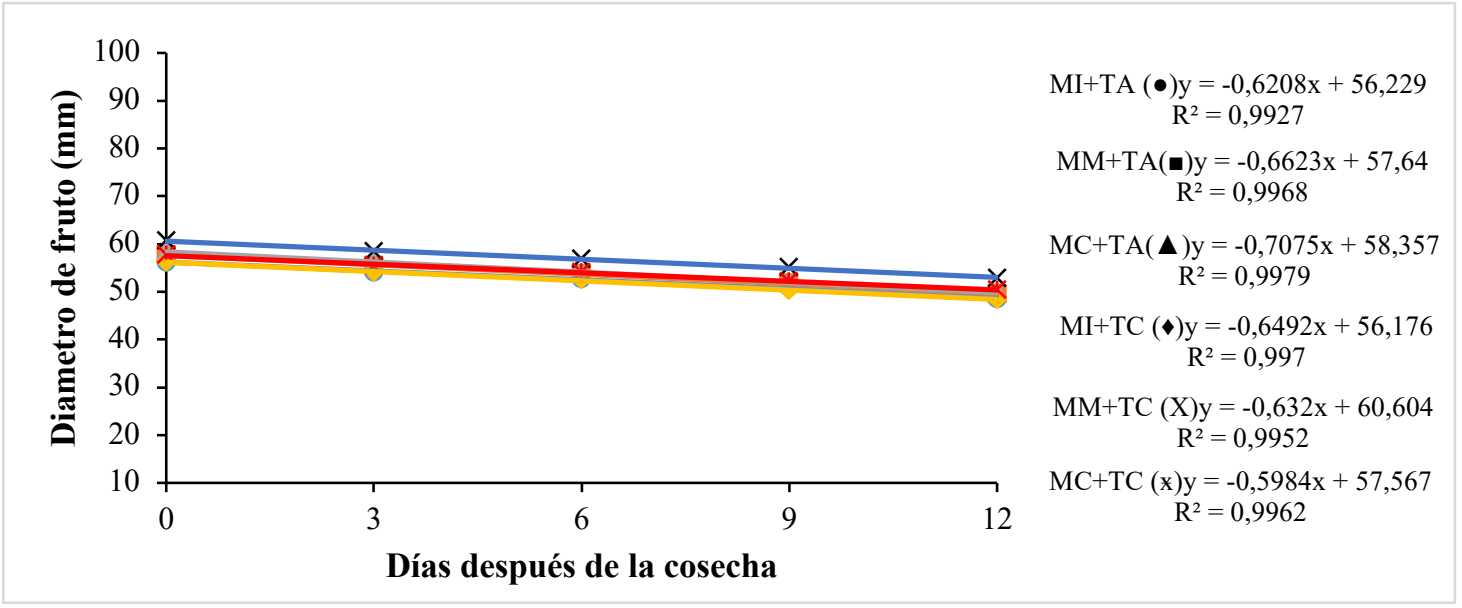

Gráfico 2. Pérdida del diámetro en frutos de pitahaya amarilla cosechados en tres estados de madurez, bajo condiciones de temperatura ambiente y controlada

Cuadro 3. Medias de diámetro de fruto $(\mathrm{mm})$ de pitahaya amarilla (Selenicereus megalanthus), letras diferentes en el día de evaluación representan separación de medias significativas de acuerdo al test de Tukey al 95\% de confianza.

\begin{tabular}{|c|c|c|c|c|c|c|}
\hline & & & & & & \\
\hline & & \multicolumn{5}{|c|}{ Diametro de fruto $(\mathrm{mm})$} \\
\hline \multicolumn{2}{|c|}{ Tratamiento } & Día 0 & Día 3 & Día 6 & Día 9 & Día 12 \\
\hline \multicolumn{2}{|c|}{$\mathrm{MI}+\mathrm{TA}$} & 56,20 & 54,15 & 52,75 & 50,91 & 48,51 \\
\hline \multicolumn{2}{|c|}{$\mathrm{MM}+\mathrm{TA}$} & 57,69 & 55,44 & 53,78 & 51,88 & 49,54 \\
\hline \multicolumn{2}{|c|}{$\mathrm{MC}+\mathrm{TA}$} & 58,54 & 56,10 & 53,92 & 52,04 & 49,96 \\
\hline \multicolumn{2}{|c|}{$\mathrm{MI}+\mathrm{TC}$} & 56,17 & 54,09 & 52,57 & 50,24 & 48,35 \\
\hline \multicolumn{2}{|c|}{$\mathrm{MM}+\mathrm{TC}$} & 60,69 & 58,44 & 56,93 & 55,14 & 52,86 \\
\hline \multicolumn{2}{|c|}{$\mathrm{MC}+\mathrm{TC}$} & 57,72 & 55,49 & 54,11 & 52,15 & 50,41 \\
\hline \multicolumn{2}{|c|}{ Probabilidad } & $0,0856 \mathrm{NS}$ & $0,1072 \mathrm{NS}$ & $0,1241 \mathrm{NS}$ & $0,0651 \mathrm{NS}$ & $0,1073 \mathrm{NS}$ \\
\hline Factores & Niveles & & & & & \\
\hline \multirow{3}{*}{$\begin{array}{c}\text { Estados de } \\
\text { madurez (A) }\end{array}$} & Inicial & $56,18 \mathrm{~b}$ & 54,12 & 52,66 & $50,57 \mathrm{~b}$ & 48,43 \\
\hline & Medio & 59,19 a & 56,94 & 55,36 & $53,51 \mathrm{a}$ & 51,20 \\
\hline & Completo & $58,13 \mathrm{ab}$ & 55,79 & 54,02 & $52,09 \mathrm{ab}$ & 50,18 \\
\hline \multirow{2}{*}{$\begin{array}{c}\text { Temperatura } \\
\text { (B) }\end{array}$} & Ambiente & 57,48 & 55,23 & 53,49 & 51,61 & 49,34 \\
\hline & Controlada & 58,19 & 56,00 & 54,54 & 52,51 & 50,54 \\
\hline \multirow{2}{*}{ Probabilidad } & Factor A & $0,0468^{*}$ & $0,0625 \mathrm{NS}$ & $0,0789 \mathrm{NS}$ & $0,0484 *$ & $0,0721 \mathrm{NS}$ \\
\hline & Factor B & $0,452 \mathrm{NS}$ & $0,4085 \mathrm{NS}$ & $0,2658 \mathrm{NS}$ & $0,3273 \mathrm{NS}$ & $0,2112 \mathrm{NS}$ \\
\hline \multicolumn{2}{|c|}{ C.V (\%) } & 3,82 & 3,92 & 4,08 & 4,05 & 4,49 \\
\hline
\end{tabular}




\section{Firmeza (NÉWTONES/ $\mathrm{cm}^{2}$ )}

En el gráfico 3, se observa que la firmeza disminuyó en el tiempo en los tres estados de madurez y temperaturas probados, donde hubo una pérdida de 0,$18 ; 0,21$ y 0,21 $\mathrm{N} / \mathrm{cm}^{2}$ en los estados de madurez inicial, media $\mathrm{y}$ completa en temperatura ambiente, respectivamente; $y$, una pérdida de 0,$33 ; 0,27$ y $0,28 \mathrm{~N} / \mathrm{cm}^{2}$ en los estados de madurez inicial, media y completa en temperatura controlada, correspondientemente (cuadro 4).
Investigación realizada por Guerrero (2014), indicó que la consistencia de los frutos se redujo durante el tiempo de almacenamiento. Resultados similares expresó García y Robayo (2008), donde el grado de maduración de la fruta, presentó una diferencia significativa tanto a $10^{\circ} \mathrm{C}$ como a $20^{\circ} \mathrm{C}$ por lo que la dureza fue disminuyendo hasta caer por debajo del $50 \%$ de su valor inicial al cabo de 20 días de almacenamiento. Serna et al. (2011) demostró que los frutos de pitahaya amarilla en estado de madurez 3 almacenados a $10 \mathrm{C}^{\circ}$, disminuyen aproximadamente $54,1 \%$ de firmeza inicial a los 15 días de almacenamiento.

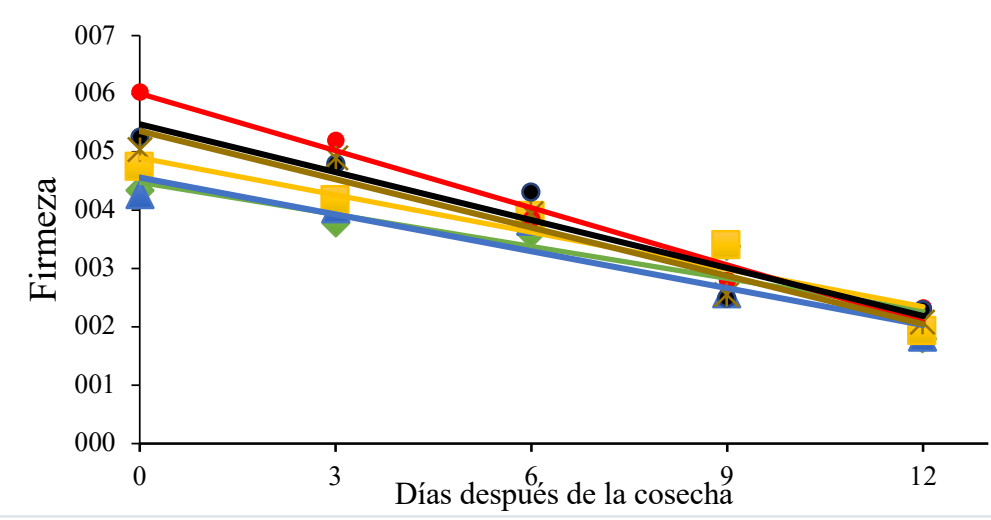

$$
\begin{aligned}
\mathrm{MI}+\mathrm{TA}(\bullet) \mathrm{y} & =-0,1833 \mathrm{x}+4,4825 \\
\mathrm{R}^{2} & =0,8294 \\
\mathrm{MM}+\mathrm{TA}(\boldsymbol{\Delta}) \mathrm{y} & =-0,2108 \mathrm{x}+4,5644 \\
\mathrm{R}^{2} & =0,9087 \\
\mathrm{MC}+\mathrm{TA}(\boldsymbol{\bullet}) \mathrm{y} & =-0,213 \mathrm{x}+4,9039 \\
\mathrm{R}^{2} & =0,9003 \\
\mathrm{MI}+\mathrm{TC}(\bullet) \mathrm{y} & =-0,3267 \mathrm{x}+6,0057 \\
\mathrm{R}^{2} & =0,9797 \\
\mathrm{MM}+\mathrm{TC}(\bullet) \mathrm{y} & =-0,2742 \mathrm{x}+5,4795 \\
\mathrm{R}^{2} & =0,9225 \\
\mathrm{MC}+\mathrm{TC}(\mathrm{x}) \mathrm{y} & =-0,2762 \mathrm{x}+5,3633 \\
\mathrm{R}^{2} & =0,9447
\end{aligned}
$$

\begin{tabular}{|c|c|c|c|c|c|c|}
\hline & & \multirow{2}{*}{\multicolumn{5}{|c|}{ Firmeza }} \\
\hline & & & & & & \\
\hline \multicolumn{2}{|c|}{ Tratamiento } & Día 0 & Día 3 & Día 6 & Día 9 & Día 12 \\
\hline \multicolumn{2}{|c|}{$\mathrm{MI}+\mathrm{TA}$} & $4,35 \mathrm{~b}$ & $3,78 \mathrm{~b}$ & 3,38 & $3,7 \mathrm{a}$ & 1,62 \\
\hline \multicolumn{2}{|c|}{$\mathrm{MM}+\mathrm{TA}$} & $4,27 \mathrm{~b}$ & $4,02 \mathrm{ab}$ & 3,80 & $2,57 \mathrm{c}$ & 1,83 \\
\hline \multicolumn{2}{|c|}{$\mathrm{MC}+\mathrm{TA}$} & $4,74 \mathrm{~b}$ & $4,17 \mathrm{ab}$ & 3,89 & $3,65 \mathrm{ab}$ & 1,93 \\
\hline \multicolumn{2}{|c|}{$\mathrm{MI}+\mathrm{TC}$} & $6,03 \mathrm{a}$ & $5,20 \mathrm{a}$ & 3,85 & $2,80 \mathrm{bc}$ & 2,33 \\
\hline \multicolumn{2}{|c|}{$\mathrm{MM}+\mathrm{TC}$} & $5,26 \mathrm{ab}$ & $4,81 \mathrm{ab}$ & 4,31 & $2,59 \mathrm{c}$ & 2,56 \\
\hline \multicolumn{2}{|c|}{$\mathrm{MC}+\mathrm{TC}$} & $5,049 \mathrm{ab}$ & $4,9 \mathrm{ab}$ & 3,95 & $2,55 \mathrm{c}$ & 2,08 \\
\hline \multicolumn{2}{|c|}{ Probabilidad } & $0,0012 *$ & $0,0168^{*}$ & $0,0825 \mathrm{NS}$ & $0,0003 *$ & $0,1516 \mathrm{NS}$ \\
\hline Factores & Niveles & & & & & \\
\hline \multirow{3}{*}{$\begin{array}{l}\text { Estados de } \\
\text { madurez (A) }\end{array}$} & Inicial & 5,19 & 4,49 & 3,62 & $3,26 \mathrm{a}$ & 1,98 \\
\hline & Medio & 4,76 & 4,41 & 4,07 & $2,58 \mathrm{~b}$ & 2,19 \\
\hline & Completo & 4,89 & 4,54 & 3,92 & $3,1 \mathrm{ab}$ & 2,01 \\
\hline \multirow{2}{*}{$\begin{array}{l}\text { Temperatura } \\
\text { (B) }\end{array}$} & Ambiente & $4,45 \mathrm{~b}$ & $3,99 \mathrm{~b}$ & 3,69 & $3,32 \mathrm{a}$ & $1,79 \mathrm{~b}$ \\
\hline & Controlada & $5,45 \mathrm{a}$ & $4,97 \mathrm{a}$ & 4,04 & $2,65 \mathrm{~b}$ & $2,3 \mathrm{a}$ \\
\hline \multirow{2}{*}{ Probabilidad } & Factor A & $0,335 \mathrm{NS}$ & $0,9065 \mathrm{NS}$ & 0,0899 NS & $0,0185^{*}$ & $0,6442 \mathrm{NS}$ \\
\hline & Factor B & 0,0004* & $0,0005^{*}$ & $0,0397^{*}$ & $0,0018^{*}$ & $0,0169^{*}$ \\
\hline \multicolumn{2}{|c|}{ C.V (\%) } & 10,33 & 13,04 & 10 & 12,95 & 24,4 \\
\hline
\end{tabular}

Gráfico 3: Pérdida de la firmeza en frutos de pitahaya amarilla cosechados en tres estados de madurez, bajo condiciones de temperatura ambiente y controlada.

Cuadro 4. Medias de firmeza de fruto de pitahaya amarilla (Selenicereus megalanthus), letras diferentes en el día de evaluación representan separación de medias significativas de acuerdo al test de Tukey al 95\% de confianza. 


\section{Sólidos solubles totales $\left({ }^{\circ}\right.$ Brix $)$}

En el gráfico 4, el comportamiento de los grados Brix en temperatura ambiente disminuyó a un ritmo de 0.02 , 0.04 y 0.03 grados Brix/día, en los frutos con madurez inicial, media y completa, respectivamente. Mientras que, en temperatura controlada la pérdida de grados Brix en madurez inicial fue 0.22 , media de 0.14 y completa de $0.01^{\circ}$ Brix (Cuadro 5).

Estos resultados coinciden con lo reportado por Jiménez et al. (2017), quienes no observaron diferencias significativas en cuanto a la concentración de sólidos solubles totales ( ${ }^{\circ} \mathrm{Brix}$ ) en los tratamientos almacenados al ambiente y al frío, ni entre los diferentes estados de madurez. Tampoco observaron una tendencia concreta, ya que existían mediciones en las que aumentaban y otras disminuían. A su vez Vásquez-Castillo et al. (2016) describió que en la variedad Palora registró mayor cantidad de sólidos solubles totales (SST) que la variedad Nacional con 20,1 y 17,9 'Brix, respectivamente; mientras que los frutos cosechados tempranamente tuvieron mayores SST que los cosechados tardíamente.

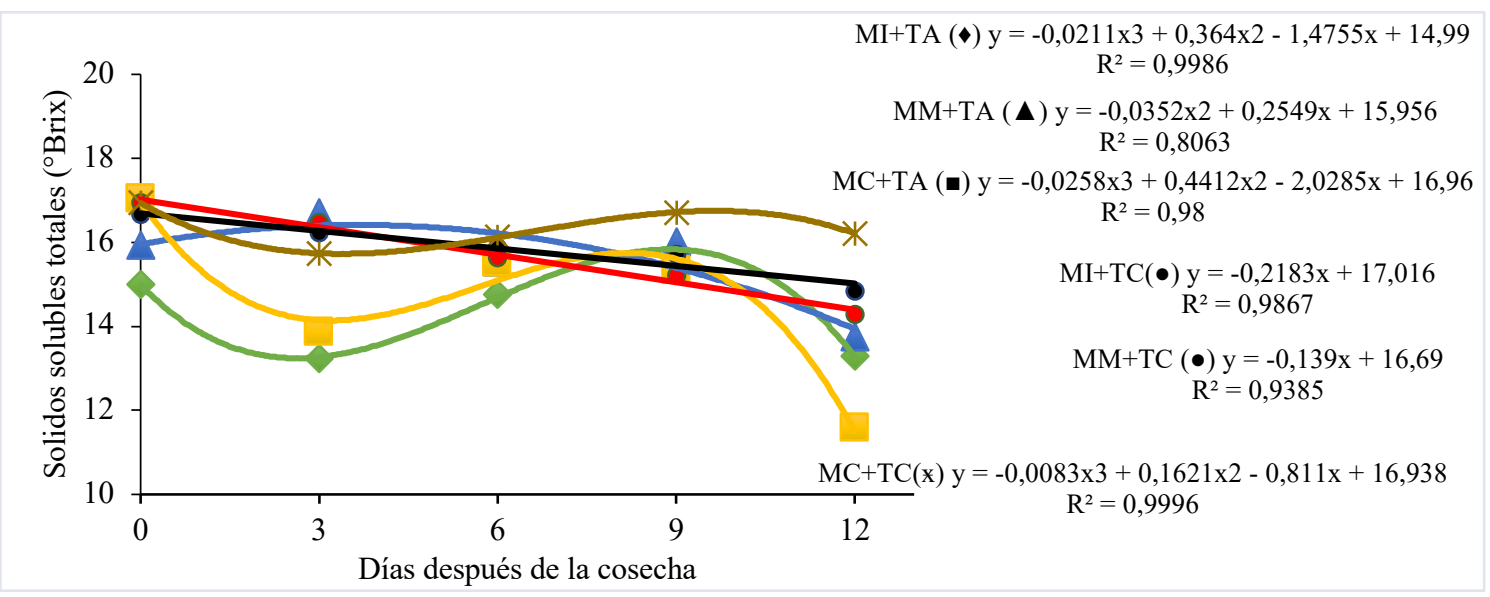

Gráfico 4: Comportamiento de ${ }^{\circ}$ Brix en frutos de pitahaya amarilla cosechados en tres estados de madurez, bajo condiciones de temperatura ambiente y controlada.

Cuadro 5 Medias de solidos solubles totales ${ }^{\circ}$ Brix de fruto de pitahaya amarilla (Selenicereus megalanthus), letras diferentes en el día de evaluación representan separación de medias significativas de acuerdo al test de Tukey al $95 \%$ de confianza.

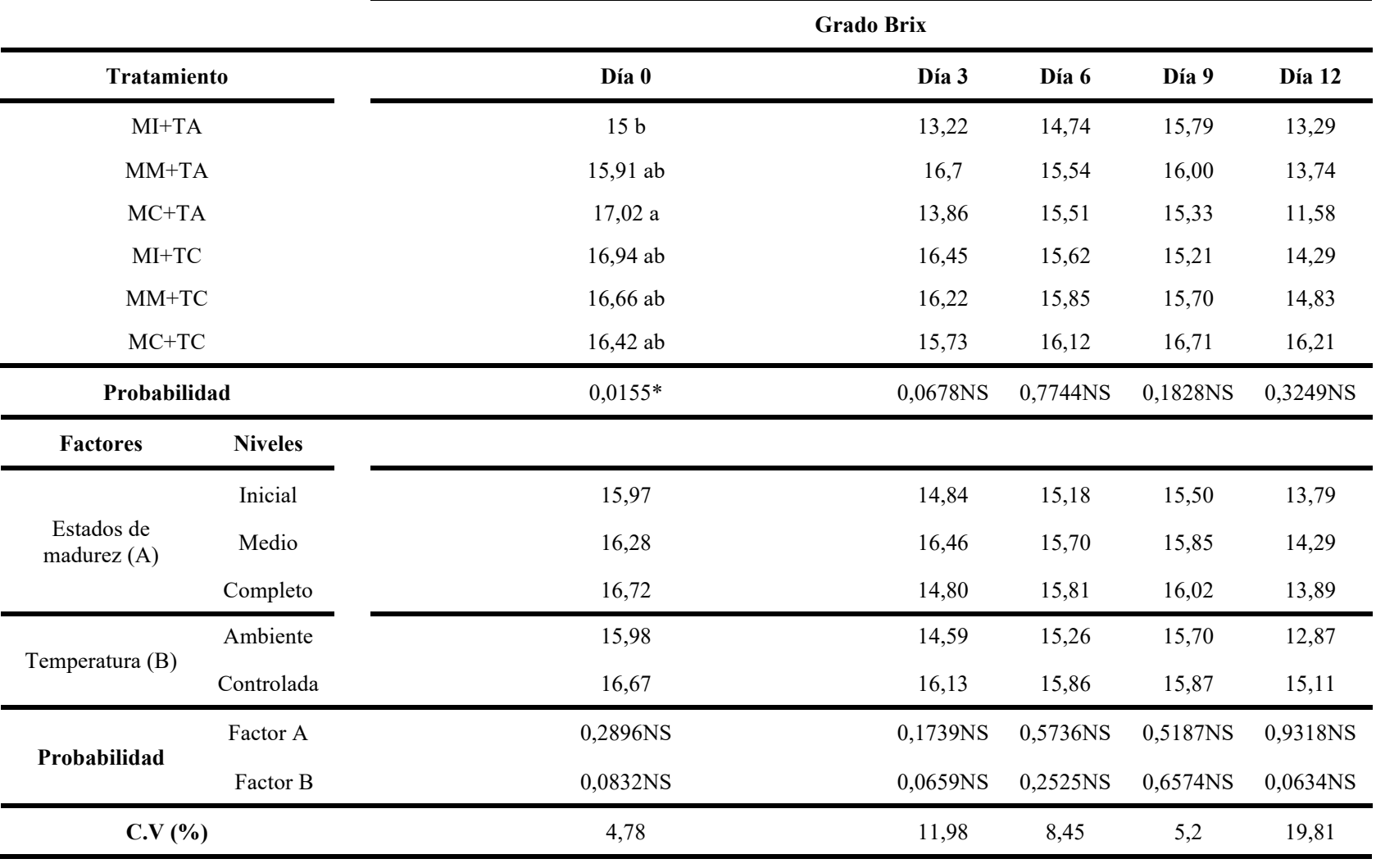




\section{pH}

El pH presentó un comportamiento ascendente en los frutos en temperatura controlada, mientras que en temperatura ambiente su comportamiento es descendente mayormente a partir del día 6 (Cuadro 6). En el gráfico 5, se observa que el pH disminuyó 0.004 , 0.02 y 0.01 en los frutos con madurez inicial, media y completa, respectivamente, en temperatura ambiente; mientras que en temperatura controlada fue de 0.04 en madurez inicial, 0.64 en madurez media y 0.03 en madurez completa.
Resultados similares presentó Sotomayor et al. (2019), quienes obtuvieron un aumento de del $\mathrm{pH}$ en los estados de madurez 0 al 6. Además, García y Robayo (2008) evidenciaron que la fruta inmadura (estado 0) obtuvo un $\mathrm{pH}$ de 4.05 , mientras que fruta madura presentó un valor de 4.7, estos datos concuerdan con los de esta investigación. A su vez Noboa (2016), quien probó el efecto de irradiación de rayo gamma sobre en la calidad postcosecha de la pitahaya amarilla, reportó que en el estado de madurez 4 alcanzó 4,86 mientras que en estado 5 de madurez obtuvo 5,06.

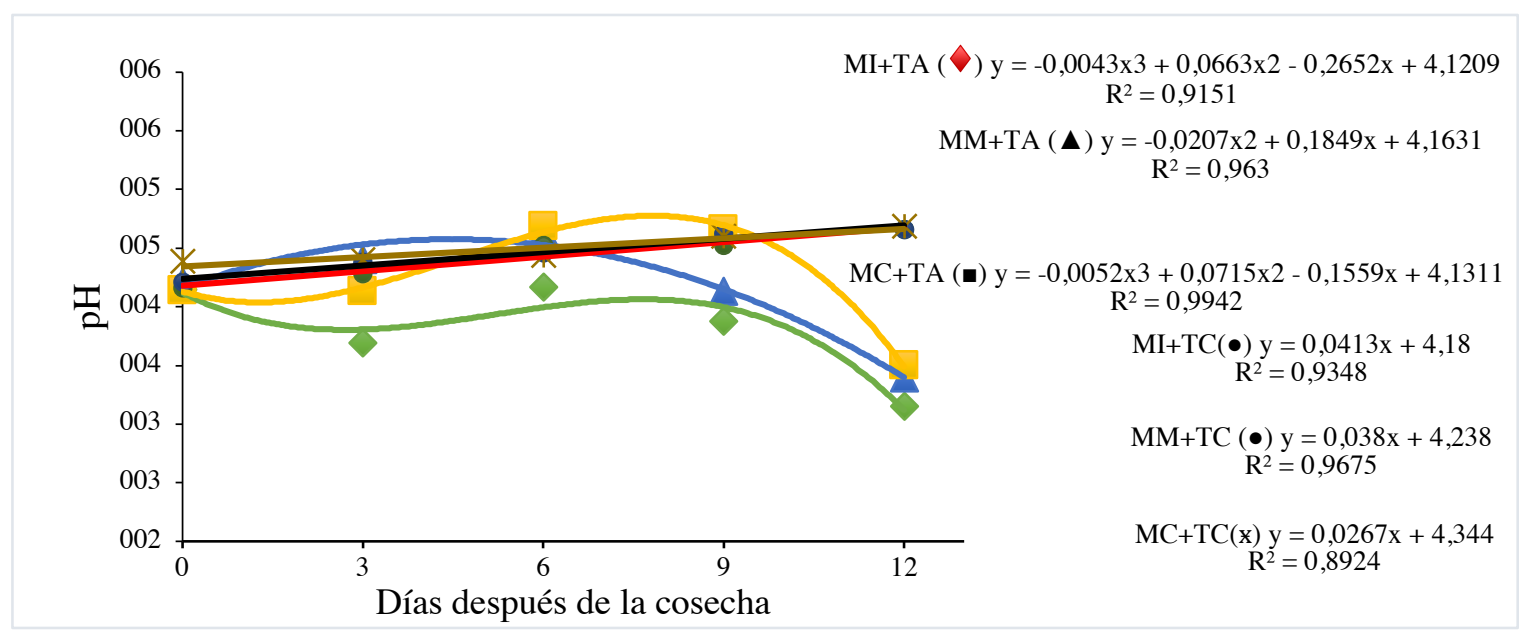

Gráfico 5. Comportamiento del $\mathrm{pH}$ en frutos de pitahaya amarilla cosechados en tres estados de madurez, bajo condiciones de temperatura ambiente y controlada.

Cuadro 6. Medias de $\mathrm{pH}$ de fruto de pitahaya amarilla (Selenicereus megalanthus), letras diferentes en el día de evaluación representan separación de medias significativas de acuerdo al test de Tukey al 95\% de confianza.

\begin{tabular}{|c|c|c|c|c|c|c|}
\hline \multicolumn{2}{|c|}{ Tratamiento } & Día 0 & Día 3 & Día 6 & Día 9 & Día 12 \\
\hline \multicolumn{2}{|c|}{$\mathrm{MI}+\mathrm{TA}$} & 4,15 & 3,68 & 4,16 & 3,87 & 3,15 \\
\hline \multicolumn{2}{|c|}{$\mathrm{MM}+\mathrm{TA}$} & 4,21 & 4,40 & 4,64 & 4,12 & 3,39 \\
\hline \multicolumn{2}{|c|}{$\mathrm{MC}+\mathrm{TA}$} & 4,14 & 4,13 & 4,68 & 4,66 & 3,49 \\
\hline \multicolumn{2}{|c|}{$\mathrm{MI}+\mathrm{TC}$} & 4,15 & 4,27 & 4,52 & 4,52 & 4,65 \\
\hline \multicolumn{2}{|c|}{$\mathrm{MM}+\mathrm{TC}$} & 4,21 & 4,37 & 4,45 & 4,62 & 4,65 \\
\hline \multicolumn{2}{|c|}{$\mathrm{MC}+\mathrm{TC}$} & 4,38 & 4,40 & 4,43 & 4,60 & 4,69 \\
\hline \multicolumn{2}{|c|}{ Probabilidad } & $0,2693 \mathrm{NS}$ & $0,2219 \mathrm{NS}$ & $0,3682 \mathrm{NS}$ & $0,5782 \mathrm{NS}$ & $0,0453 \mathrm{NS}$ \\
\hline Factores & Niveles & & & & & \\
\hline \multirow{3}{*}{$\begin{array}{l}\text { Estados de madurez } \\
\text { (A) }\end{array}$} & Inicial & 4,15 & 3,98 & 4,34 & 4,19 & 3,9 \\
\hline & Medio & 4,21 & 4,34 & 4,55 & 4,37 & 4,02 \\
\hline & Completo & 4,26 & 4,26 & 4,56 & 4,63 & 4,09 \\
\hline \multirow{2}{*}{ Temperatura (B) } & Ambiente & 4,17 & 4,07 & 4,49 & 4,22 & 3,35 \\
\hline & Controlada & 4,25 & 4,35 & 4,47 & 4,58 & 4,67 \\
\hline \multirow{2}{*}{ Probabilidad } & Factor A & $0,411 \mathrm{NS}$ & $0,1944 \mathrm{NS}$ & $0,4177 \mathrm{NS}$ & $0,4872 \mathrm{NS}$ & $0,8955 \mathrm{NS}$ \\
\hline & Factor B & $0,2293 \mathrm{NS}$ & $0,141 \mathrm{NS}$ & $0,8568 \mathrm{NS}$ & $0,2323 \mathrm{NS}$ & $0,0008^{*}$ \\
\hline \multicolumn{2}{|c|}{ C.V (\%) } & 3,71 & 10,52 & 7,64 & 16,6 & 21,65 \\
\hline
\end{tabular}




\section{Acidez titulable}

La acidez titulable del fruto presentó un comportamiento descendente en los dos tipos de temperatura y los diferentes estados de madurez. Los resultados de este análisis se consideraron a partir del día 3 del periodo de evaluación, donde los estados de madurez inicial y media en temperatura controlada alcanzaron la mayor acidez durante los tiempos evaluados (Cuadro 7). En el gráfico 6, se observa que la acidez en temperatura ambiente disminuyó en $0.0008,0.0005$ y 0.0008 en los frutos con madurez inicial, media y completa, respectivamente; mientras que en temperatura controlada la reducción fue de 0.22 en madurez inicial, 0.14 en madurez media y 0.0001 en madurez completa.

Estos resultados concuerdan por los presentado por Sotomayor et al. (2019), quienes demostraron que la acidez titulable disminuye con los estados de madurez y el tiempo de almacenamiento.
Mientras que Rodríguez et al. (2005) obtuvieron resultados que difieren con esta investigación, los cuales demuestran que la acidez titulable en el grado de madurez de cosecha hasta el día 9 del almacenamiento el cual fue más evidente, mientras que la temperatura no mostró diferencias significativas. A su vez Dueñas et al. (2008) mencionó que frutos almacenados a $18^{\circ} \mathrm{C}$ tienen una tendencia hacia la disminución en la acidez total titulable (ATT) bastante pronunciada durante los primeros 3 días de almacenamiento donde el mayor cambio se observó durante los primeros días y está relacionado con la alta respiración que manifiestan los frutos de pitahaya.Yi-Lu-J et al. (2011) encontró que la acidez titulable expresada como ácido cítrico se redujo durante el almacenamiento en frutas en grado de maduración 4 , presentado valores más bajos de acidez titulable en referencia a los tipos de temperaturas de almacenamiento probado

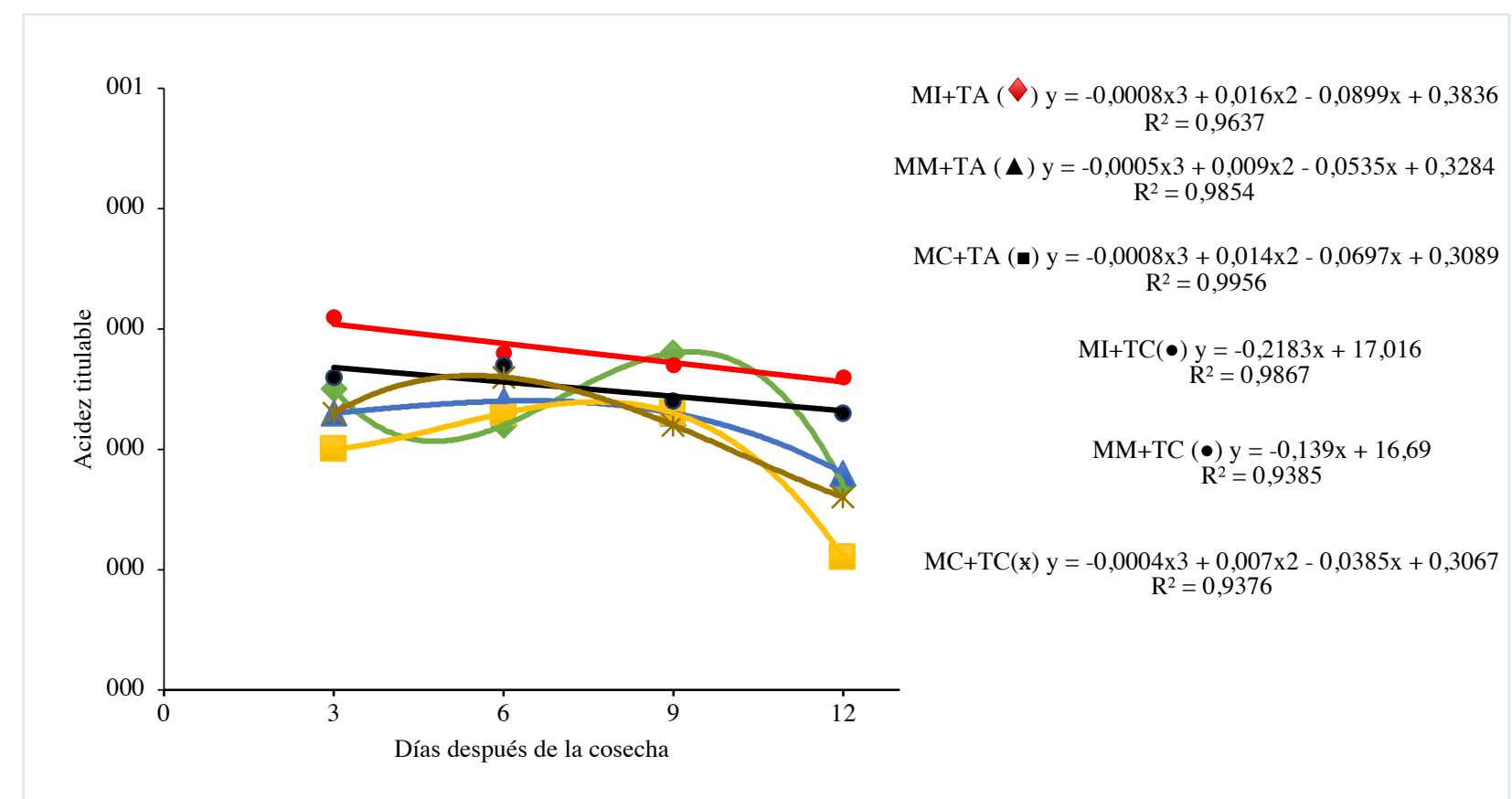

Gráfico 6: Comportamiento de la acidez titulable en frutos de pitahaya amarilla cosechados en tres estados de madurez, bajo condiciones de temperatura ambiente y controlada. 
Cuadro 7. Medias de acidez titulable de fruto de pitahaya amarilla (Selenicereus megalanthus), letras diferentes en el día de evaluación representan separación de medias significativas de acuerdo al test de Tukey al 95\% de confianza.

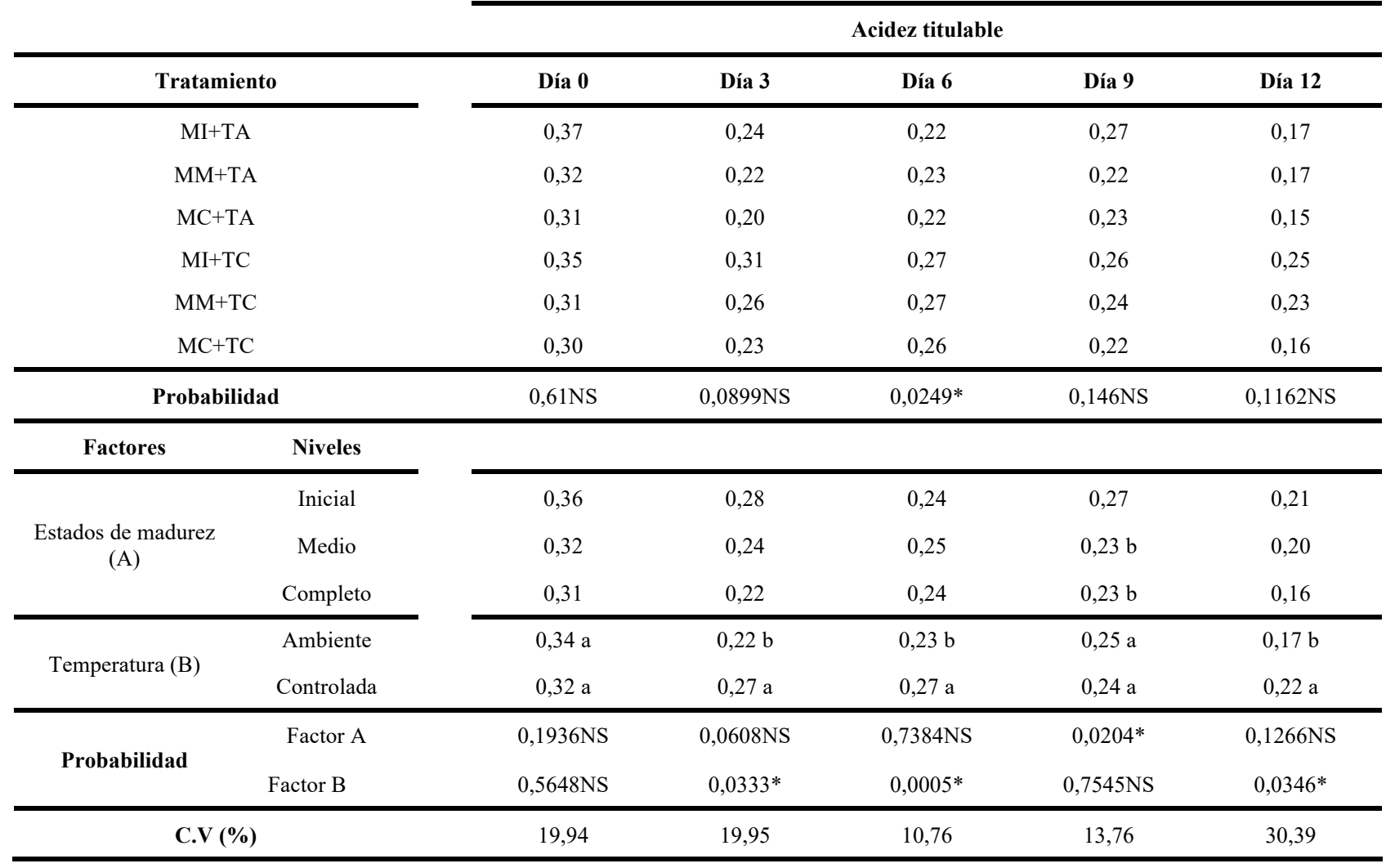

\section{CONCLUSIONES}

Los factores de madurez y temperatura no influyeron estadísticamente en los diferentes tratamientos evaluados, pero los frutos en los diferentes estados de madurez inicial, media y completa a temperatura ambiente y controlada presentaron disminución en la variable peso, diámetro y firmeza.

El estado de madurez inicial a temperatura controlada presentó la menor reducción de peso, $\mathrm{pH}$ y mayor reducción de la acidez y firmeza a excepción del diámetro y sólidos solubles que presentaron mejores resultados en los frutos cosechados a madurez media en temperatura controlada durante el periodo de evaluación.

\section{AGRADECIMIENTOS}

Los autores agradecen a la Hacienda El Okaso, a Don Olvin Intriago, Ing. Katty Ormaza y Afranio Esmeralda, por el apoyo técnico y logístico brindado en la presente investigación.

\section{LITERATURA CITADA}

Aguilar, G. 2015. Evaluación de tres enraizantes y dos tamaños de cladodios en la propagación asexual de pitahaya amarilla Cereus triangularis (L.) Haw., en Yantzaza. Tesis. Ing. Agrónomo. Universidad Nacional de Loja. Loja, EC. p 116.

Alvarado, J. 2014. Caracterización postcosecha de la calidad del Fruto de pitahaya amarilla (Selenicereus megalanthus) y Roja (Hylocereus undatus). Tesis. Ing. Agrónomo. Universidad de Guayaquil. Guayas, EC. p 80.

Balderas, V., Palafox, L., Castro, AS., Saucedo, C. 2016. Evaluación de las propiedades físicas y calidad organoléptica y nutricional de frutos de pitaya (Stenocereus pruinosus). Rev. Iberoam Cienc. 3(7):8692.

Dueñas, G., Narváez, C., Restrepo, S. 2008. Inhibición de lesiones por frío de pitaya amarilla (Acanthocereus pitajaya) a través del choque térmico: catalasa, peroxidasa y polifenoloxidasa. Acta Biol. Colomb. 13:95-106.

Dueñas, Y., Narváez, C., Restrepo, S. 2009. El choque térmico mejora la aptitud al almacenamiento refrigerado de pitaya amarilla. Rev. Agronomía Colombiana. 27 (1): 105-110.

García, M., Robayo, P. 2008. Evaluación del uso de atmósferas modificadas pasivas y temperaturas bajas en la conservación de pitaya amarilla (Selinecereus 
megalanthus Shuman). Rev. Corpoica Ciencia y Tecnología Agropecuaria, 9 (1):30-39.

Guerrero, M. 2014. Estudio del manejo postcosecha de pitahaya amarilla (Selenecereus megalanthus) procedente del Cantón Pedro Vicente Maldonado de la Provincia de Pichincha. Tesis. Ing. Agroindustrial. Escuela Politécnica Nacional. Quito- Pichincha, EC. p 42.

Huachi, L., Yugsi, E., Paredes, M., Verdugo, K., Coba, P. 2015. Desarrollo de la pitahaya (Cereus SP.) en Ecuador. Rev. La Granja, 22 (2): 50-58.

Jiménez, L., González, M., Cruz, S., Santana, R., Villacís L. 2017. Análisis postcosecha de frutos de pitahaya amarilla (Cereus triangularis Haw), a distintos nivel.es de madurez y temperatura. Rev. Selva Andina Biosph. 5 (2):107-115.

Jiménez, L., González, M., Yanez, A., Cruz, S., Villacís L. 2017. Características organolépticas de frutas de pitahaya amarilla (Cereus triangularis Haw.) bajo dos condiciones de almacenamiento. Rev. Selva Andina Biosph. 5 (2): 160-167.

Montesinos, J., Rodríguez, L., Ortiz, R., Fonseca, M., Ruiz, G., Guevara, F. 2015. Revisión bibliográfica pitahaya (Hylocereus spp.) un recurso fitogenético con historia y futuro para el trópico seco mexicano. Rev. INCA, 36, 67-76.

Magaña, B., Baldín A., Corrales G., Saucedo V., Sauri, E. 2013. Variaciones bioquímicas-físiológicas y físicas de las frutas de pitahaya (Hylocereus undanatus) almacenadas en ambiente natural. Rev Iber Tecnología Postcosecha. 4(1):21-30

NTE-INEN-025. 2005. Frutas frescas. Pitahaya amarilla. Requisitos. Norma Tecnica Ecuatoriaana, 1: 1-10.
Noboa, A. 2016. Estudio del efecto de irradiación con rayos gamma en la calidad postcosecha de la pitahaya amarilla (Selenicereus megalanthus) entera y cortada. Tesis. Ing. Química. Escuela Politécnica Nacional. Quito, EC. p 229.

Rodríguez, D., Patiño, M., Miranda, D., Fischer, G., y Galvis, J. 2005. Efecto de dos índices de madurez y dos temperaturas de almacenamiento sobre el comportamiento en almacenamiento sobre el comportamiento en postcosecha de la pitahaya amarilla (Selenicereus megalanthus Haw.). Rev. Fac. Nal. Agr. Medellín, 58 (2), 2827-2

Serna, L., Torres, L., Ayala, A. 2011. Aplicación de 1metilciclopropeno, una alternativa A la estacionalidad de la pitahaya amarilla. Revista Alimentos Hoy, 20 (23). 3-20.

Sotomayor, A., Pitizaca, S., Sánchez, M., Burbano, A., Díaz, A., Otros. 2019. Evaluación físico química de fruta de pitahaya (Selenecereus megalanthus) en diferentes estados de desarrollo. Rev. Scielo. 10 (1): $1039-6542$

Vargas, Y., Pico, J., Díaz, A., Sotomayor, D., Burbano, A., Caicedo, C., Paredes, N., Congo, C., Tinoco, L., Bastidas, S., Chuquimarca, J., Macas, J., Viera, W. 2020. Manual del Cultivo de Pitahaya para la Amazonía Ecuatoriana. Los Sachas - Ecuador: Estación Experimental de la Amazonia.

Vásquez-Castillo, W., Aguilar, K., Vilaplana, R., Viteri, P., Viera, W., Valencia, S. 2016. Calidad del fruto y perdidas postcosecha de pitahaya amarilla (Stenocereus megalanthus), en Ecuador. Rev. Agronomía Colombiana. 34 (1):1-3.

Yi-Lu-J., Tzong, L., Ching, L. 2011. Phenology, canopy composition, and fruit quality of yellow pithaya in tropical Taiwan. Rev. HortScience, 46(11), 1497-1502. 